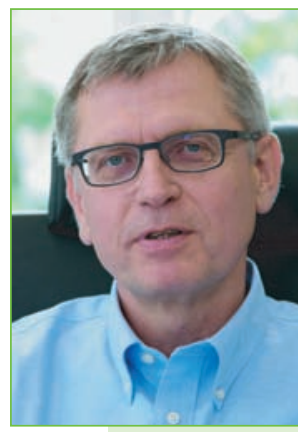

\title{
Demenz - auch eine Domäne der Phytotherapie?
}

Erreichte in früheren Jahrhunderten ein Mensch ein hohes Alter, womöglich mehr als 80 Jahre, so sprach man bewundernd von einem gesegneten Alter. Zwischenzeitlich hat sich in zahlreichen Gesellschaften ein tiefgreifender demografischer Wandel vollzogen, der dadurch gekennzeichnet ist, dass Langlebigkeit nicht mehr die Ausnahme, sondern die Regel ist. Mit der Zunahme der Weltbevölkerung einerseits und einer überproportionalen Zunahme Älterer und Hochbetagter andererseits erreichen altersassoziierte Erkrankungen eine bisher nicht gekannte epidemiologische Tragweite. Dies trifft insbesondere für die Demenz zu, eine typische Erkrankung des Alters. Trotz mehr als zwei Jahrzehnten intensivierter Forschung liegen die Ursachen demenzieller Erkrankungen, insbesondere der Alzheimer-Krankheit, noch weitgehend im Dunkeln. Dementsprechend sind Angriffspunkte kausaler Therapien oder Präventionsansätze nach wie vor Gegenstand von Hypothesen (1). Bemühungen, durch Eingriffe in die lange als kausal vermutete Amyloidpathologie den Verlauf der Krankheit zu verlangsamen und ihre Symptomatik zu beeinflussen, haben die in sie gesetzten Hoffnungen bisher nicht erfüllt (2). In dieser Situation kommt bis auf Weiteres der symptomatischen Behandlung, vorzugsweise in frühesten Stadien der demenziellen Erkrankung beginnend, größte Bedeutung zu.

Vom Bundesinstitut für Arzneimittel und Medizinprodukte (BfArM) zugelassen zur symptomatischen Behandlung der Alzheimer-Krankheit ist neben Cholinesterasehemmern und einem nichtkompetitiven NMDA-Antagonisten (Memantine) auch ein Phytopharmakon: monografiekonformer Ginkgobiloba-Extrakt. Dieser ist darüber hinaus zur symptomatischen Behandlung der vaskulären Demenz und Mischformen zugelassen. Er ist als eines von wenigen pflanzlichen Medikamenten als Standardtherapeutikum zur Behandlung einer schweren Erkrankung eingestuft und damit nach der Ausnahmeliste zu SGB V § 34 auf Kosten der gesetzlichen
Krankenversicherung verordnungsfähig. Das Institut für Qualität und Wirtschaftlichkeit im Gesundheitswesen (IQWiG) hat dem monografiekonformen Ginkgo-biloba-Extrakt einen patientenrelevanten Nutzen in der Behandlung der Alzheimer-Demenz bescheinigt (3). (Die Nutzenbewertung für die Behandlung vaskulärer Demenzen war bisher noch nicht Gegenstand von IQWiG-Verfahren.) Alle Studien, die den Qualitätsanforderungen des IQWiG genügten, waren mit dem Spezialextrakt EGb $761^{\circledR}$ durchgeführt worden. Weitere Pflanzenextrakte mit für demenzielle Erkrankungen interessantem Wirkprofil befinden sich in frühen Phasen der Forschung.

Dies ist insofern bemerkenswert, als nun ein Extrakt, ein - wie für Phytopharmaka typisch - Mehrstoffgemisch mit multifaktoriellem Ansatz, hohe Anforderungen überwunden hat und der allgemeinen Therapie dieser schwerwiegenden Erkrankung zur Verfügung steht. Dies sollte uns Ansporn sein, weiter mit adäquaten Methoden den Wirkmechanismus sowie Wirksamkeit, Verträglichkeit und Sicherheit von Phytopharmaka zu erforschen.

Hans-Peter Volz

Krankenhaus für Psychiatrie, Psychotherapie und Psychosomatische Medizin Schloss Werneck

\section{LITERATUR}

1 Daviglus ML, Bell CC, Berrettini W et al. National Institutes of Health State-of-the-Science Conference Statement: Preventing Alzheimer's Disease and Cognitive Decline. NIH Consens State Sci Statements 2010; 27: $1-30$

2 Holtzman JL. Is the dementia of Alzheimer's disease due to the toxicity of $\beta$-amyloid or tau? The implications of this question for drug discovery. J Nutr Health Aging 2012; 16: 795

3 IQWiG. Ginkgohaltige Präparate bei Alzheimer Demenz. Abschlussbericht. Köln: IQWiG; 2008 